\title{
Ketamine perturbs perception of the flow of time in healthy volunteers
}

\author{
Jennifer T Coull • Hannah Morgan • Victoria C Cambridge • James W Moore • \\ Francesco Giorlando • Ram Adapa • Philip R. Corlett • Paul C Fletcher
}

Received: 16 February 2011 / Accepted: 4 May 2011 /Published online: 21 May 2011

(C) The Author(s) 2011. This article is published with open access at Springerlink.com

\begin{abstract}
Rationale Disturbances in the subjective experience of time have been observed both in schizophrenia and following acute administration of ketamine. However, effects of ketamine on more objective timing tasks have not yet been measured in humans, nor has it been established that timing effects are not merely secondary to a more general dysfunction in working memory (WM).

Objective and methods In a double-blind placebocontrolled crossover study, we characterised the effects of ketamine $(100 \mathrm{ng} / \mathrm{ml}$ blood plasma level) on performance of perceptual timing and colour discrimination tasks, which were matched for WM and attentional demands. To test the ubiquity of ketamine's effects on timing, we also examined two distinct measures of temporal predictability.
\end{abstract}

Jennifer T Coull and Hannah Morgan are joint first authors.

J. T. Coull $(\bowtie)$

Laboratoire de Neurobiologie de la Cognition,

Université de Provence \& CNRS,

Pôle 3C, 3 Place Victor Hugo,

13331 Marseille Cedex 3, France

e-mail: jennifer.coull@univ-provence.fr

H. Morgan • V. C. Cambridge · J. W. Moore - F. Giorlando •

R. Adapa $\cdot$ P. C. Fletcher

Department of Psychiatry, Brain Mapping Unit,

University of Cambridge,

Cambridge, UK

P. R. Corlett

Department of Psychiatry, Connecticut Mental Health Center,

Yale University,

New Haven, CT, USA

F. Giorlando

Department of Clinical and Biomedical Sciences, University of

Melbourne,

Melbourne, VIC, Australia
Results Ketamine significantly distorted the subjective experience of time as measured by the ClinicianAdministered Dissociative States Scales. Critically, ketamine also impaired accuracy on the perceptual timing task while having no effect on performance of the colour perception task. Although ketamine did not impair the ability to use prelearned temporal (or spatial) cues to predict target onset (or location), it did slow reaction times at long delays following non-informative neutral cues, suggesting an impaired ability to use the unidirectional flow of time itself to make temporal predictions.

Conclusions Ketamine induced selective impairments in timing, which could not be explained by more fundamental effects on the ability to hold information in WM. Rather our collected findings suggest that ketamine may disturb timing by selectively impairing the way in which information is temporally manipulated within WM.

Keywords Ketamine - Timing · Duration - NMDA · Glutamate $\cdot$ Temporal expectation $\cdot$ Hazard function . Foreperiod $\cdot$ Temporal orienting $\cdot$ Attention

\section{Introduction}

The glutamatergic $N$-methyl-D-aspartate (NMDA) receptor antagonist ketamine is a useful pharmacological model of psychosis (Krystal et al. 1994; Corlett et al. 2007a). In healthy participants, it induces perceptual changes (Krystal et al. 1994; Duncan et al. 2001; Stone and Pilowsky 2006), thought disorder (Krystal et al. 2005; Malhotra et al. 1996; Duncan et al. 2001) and negative symptoms (Krystal et al. 2005; Duncan et al. 2001), as well as delusion-like thinking (Pomarol-Clotet et al. 2006). Moreover, ketamine induces selective impairments in cognitive function (Morgan and 
Curran 2006) that are comparable to those observed in schizophrenia (Fletcher and Honey 2006). Notably, both ketamine (Honey et al. 2003, 2004) and schizophrenia (Perry et al. 2001; Kim et al. 2004) are associated with difficulties in manipulating, rather than maintaining, information in working memory (WM). While ketamine does not reproduce the signs and symptoms of established schizophrenia, it has been argued that the perceptual and cognitive changes it induces resemble those found during prodromal stages of the illness (Pomarol-Clotet et al. 2006; Corlett et al. 2006, 2007a, 2007b, 2011).

One feature of schizophrenia that has not been wellexplored under ketamine is that of the experience of time. Numerous studies have demonstrated that patients with schizophrenia show reduced accuracy in estimating periods of time in the minutes-to-hours range (Rabin 1957; Johnson and Petzel 1971) and in timing of stimulus durations in the range of several hundreds of milliseconds to a few seconds (Lhamon and Goldstone 1956; Tysk 1983; Elvevåg et al. 2003; Davalos et al. 2003; Carroll et al. 2008, 2009). However, the effects of ketamine on timing are uncertain: in rats, ketamine had no direct effect on timing (Cheng et al. 2006) but instead 'unlocked' the clock-speeding effects of cocaine that had otherwise been masked by extensive training (Cheng et al. 2007a). Yet, subjective reports in healthy volunteers indicate that ketamine can distort the experience of time (Pomarol-Clotet et al. 2006; Stone and Pilowsky 2006), a phenomenon also observed in schizophrenia (Freedman 1974; Seeman 1976; Vogeley and Kupke 2007).

Although the effects of ketamine on timing have not yet been explored experimentally in humans, Rammsayer (2006) noted that memantine (a lower-affinity NMDA antagonist) impaired timing of auditory stimuli in the seconds $(\sim 1 \mathrm{~s}$.) but not tens of milliseconds $(\sim 50 \mathrm{~ms})$ range. Timing in the range of seconds, but not milliseconds, requires support from WM and sustained attention processes (Gibbon et al. 1984; Zakay and Block 1996): a secondsrange stimulus must be continually attended from stimulus onset to offset (as opposed to estimates of, e.g. pitch or colour, which can be estimated almost instantaneously), and the contents of WM must be continuously updated as stimulus presentation unfolds. Given the pattern of memantine's effects, Rammsayer (2006) concluded that its selective effects on seconds-range timing were secondary to its effects on WM. Given differences in their pharmacological specificity, we cannot presume that ketamine's effects would be similar to those of memantine nor, from a more cognitive point of view, that such effects necessarily reflect a more fundamental effect on WM. Timing of longer, seconds-range durations depends not only upon WM but also, of course, upon an index of elapsed time itself, for example accumulation of temporal pulses (Gibbon et al. 1984; Treisman 1963) or temporal integra- tion of steadily climbing neuronal activity (Reutimann et al. 2004). Critically, psychophysical evidence suggests that this timing mechanism may be quite distinct for millisecond- and seconds-range timing (Gibbon et al. 1997; Buonomano et al. 2009). Thus, it is possible that a deficit in the seconds but not milliseconds, range reflects a specific temporal, rather than WM, deficit. We therefore sought to both establish whether ketamine, like memantine, is associated with a disturbance in an objective measure of time perception in the seconds time range and, crucially, to dissociate such an effect from concurrent mnemonic disturbance by directly comparing the effects of ketamine on performance of timing and colour discrimination tasks that were matched for WM demands (Coull et al. 2004, 2008). A ketamine-induced impairment of the timing, but not colour, task would strongly suggest a selective timing impairment over and above any effects on WM.

However, 'timing' is an imprecise term (Michon 1985; Pöppel 1997; Coull and Nobre 2008) that can refer equally well to how long a current event lasts (duration estimation) or when a future event is likely to occur (temporal prediction). Since ketamine has previously been shown to modulate neural indices of prediction error (e.g. Corlett et al. 2007a; 2011), we examine performance not only on time perception but also on measures of temporal prediction. Specifically, we tested the impact of ketamine on two distinct indices of temporal prediction derived from the temporal-orienting paradigm (Coull and Nobre 1998; Nobre 2001), a temporal analogue of the classic Posner covert orienting of spatial attention task (Posner et al. 1980). In the task, visual cues provide valid, invalid or neutral information concerning the likely delay before an imminent target is presented. The behavioural benefit of temporally informative cues is indexed by faster reaction times during valid compared to invalid trials (Coull and Nobre 1998; Griffin et al. 2001). Yet, even when the onset of the target cannot be predicted by temporal cues (i.e. in the neutral condition), the unidirectional flow of time itself, or 'time's arrow' (Eddington 1928), provides a degree of predictive power: since time flows inexorably forward an event that we expect to occur, but has not yet occurred, must do so at some time in the future. The 'hazard function' measures the increasing conditional probability (and, hence, increasing sense of temporal expectation) over time that an event will occur, given that it has not already occurred (Elithorn and Lawrence 1955; Luce 1986). Increasing temporal certainty of target presentation over time allows the response plan to be updated dynamically, as the trial unfolds, which ultimately translates into faster RTs at longer delays (Niemi and Näätänen 1981). The orienting paradigm can therefore index temporally predictive information that is conveyed either by valid, prelearned cues (temporal condition) or by the very passage of time itself (neutral condition). In order 
to control for possible, though unlikely (Morgan and Curran 2006), effects of ketamine on non-specific attentional processes, we also examined effects of ketamine on the more classic, spatial, version of the task.

\section{Methods}

\section{Participants}

We collected data from 13 (6 male and 7 female; mean age 23.1 years) right-handed, healthy volunteers. Although three further volunteers had been recruited, their data could not be included in the final analysis due either to technical difficulties or subject dropout. Research was approved by Addenbrookes NHS Trust Research Ethics Committee. Participants spoke fluent English, had normal or corrected-to-normal vision with no colour blindness, and reported no history of clinical drug or alcohol abuse or of psychiatric illness.

\section{Experimental design}

Each participant was tested on two occasions, with a minimum of 1 week between sessions. A within-subject, double-blind crossover design was used such that participants received ketamine in one session and placebo in the other. Participants also completed two learning tasks during the testing session, and performed all tasks in the same order from one session to the next. Here, we report data for the two timing tasks (data for other tasks will be reported elsewhere).

Ketamine infusion protocol

Racemic ketamine ( $2 \mathrm{mg} / \mathrm{nl}$ solution) or saline solution was administered intravenously by a computerised targetcontrolled infusion system, which calculated infusion rates necessary to achieve the 'target' blood concentration: $100 \mathrm{ng} / \mathrm{ml}$ plasma for $75 \mathrm{~min}$ immediately followed by a further $40 \mathrm{~min}$ at $200 \mathrm{ng} / \mathrm{ml}$ plasma. The timing tasks reported here were performed during the $100 \mathrm{ng} / \mathrm{ml}$ infusion period only. The infusion pump (Graseby 3500, Smiths Medical, Ashford, UK) was controlled by a personal laptop computer running Stanpump software, programmed to use a two-compartmental pharmacokinetic model for ketamine (Absalom et al. 2007) to calculate infusion rates. The order of infusion was counterbalanced across participants.

\section{Cognitive tasks}

Both tasks were performed at $100 \mathrm{ng} / \mathrm{ml}$ blood plasma level of ketamine. Twelve participants completed the discrimination tasks and 11 completed the orienting tasks.

\section{Temporal and colour discrimination}

Task The trial structure and stimuli used in the temporal and colour tasks were identical (Fig. 1), with the only

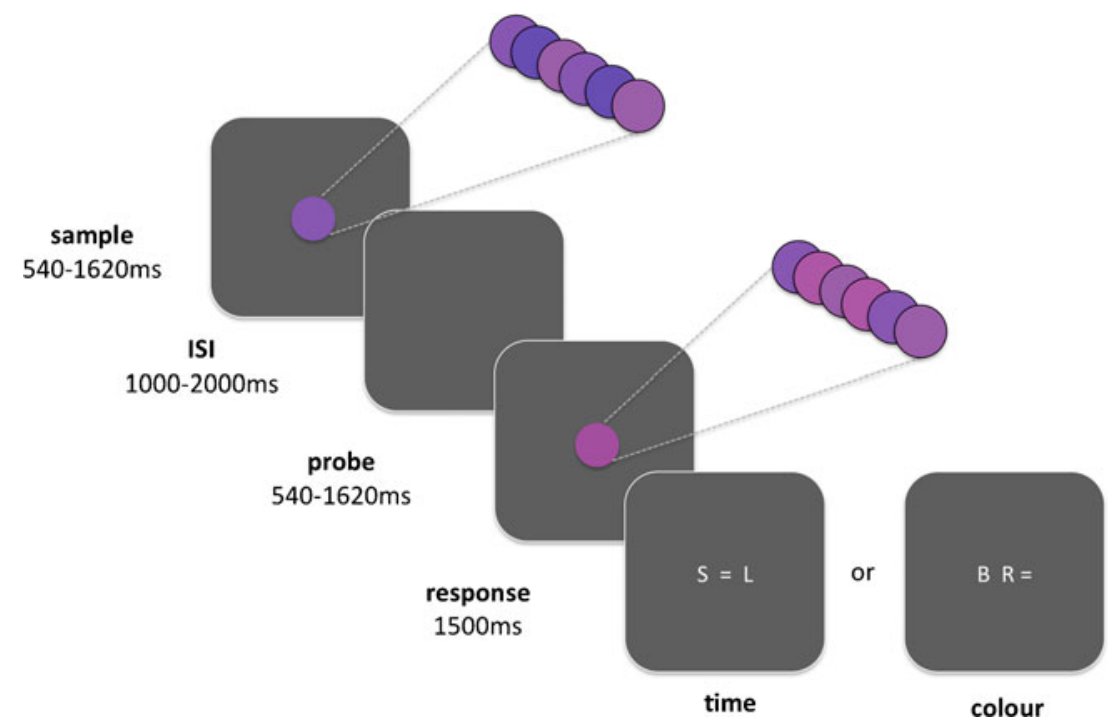

Fig. 1 Temporal and colour discrimination task. Participants estimated either the duration or colour of two consecutive stimuli. The first (sample) and second (probe) stimuli were presented for one of three durations $(540,1,080,1,620 \mathrm{~ms})$ and had an overall percept of one of three shades of purple (maroon, violet or indigo). Stimuli were not of a uniform colour but instead comprised rapidly flickering $(90 \mathrm{~ms})$ presentations of three of five different shades of purple (see inset) to give the overall colour percept. Participants indicated whether the probe was shorter $(S)$, longer $(L)$ or the same $(=)$ duration as the sample ('time' condition) or redder $(R)$, bluer $(B)$ or the same $(=)$ shade of purple as the sample ('colour' condition) using a three-choice button press at the onset of the response screen 
difference being whether participants were asked to make time or colour judgements. In the time condition, participants estimated whether the duration of the second (probe) stimulus was shorter (S), equal to (=), or longer (L) than the previous (sample) stimulus. In the colour condition, participants estimated whether the probe was redder $(\mathrm{R})$, equal to $(=)$, or bluer (B) than the sample. The response screen presented three alphanumeric characters, indicating each of the three possible responses for each condition. Their spatial position could vary on a trial-by-trial basis. Participants pressed one of the three corresponding response buttons (left, middle or right) to indicate their estimate of relative duration or colour. The response screen appeared for 1,500 ms, during which time the subject gave their response, and any responses slower than $1,500 \mathrm{~ms}$ were not recorded. Inter-trial intervals varied pseudorandomly from 1 to $2 \mathrm{~s}$. Participants performed 36 trials per condition, which comprised equal numbers of trials in which the probe was shorter than/longer than/equal to the sample in the time condition, or bluer than/redder than/ equal to the sample in the colour task. The time and colour conditions were presented twice each in a blocked manner, with each block comprising 18 trials of either the time condition or the colour condition.

Stimuli The sample and probe stimuli were presented consecutively, separated by a pseudo-random interstimulus interval of 1-2 s. Each stimulus was presented for one of three durations (540,1,080 or 1,620 ms), and had an overall percept of one of three shades of purple (maroon, violet or indigo) ranging from a reddish to a bluish hue. Duration and colour stimulus attributes were counterbalanced such that any of the three stimulus durations could be paired with any of the three colours. The stimuli to be estimated were not a uniform colour for the entire duration of stimulus presentation. Rather, rapidly alternating presentations $(90 \mathrm{~ms})$ of five different shades of purple across the entire stimulus duration gave an overall percept of maroon, violet or indigo. During the colour task, the participant estimated the average shade of purple by amalgamating all shades presented during the flickering percept. This rather unusual colour task was designed to ensure that subjects maintained attention, and continually updated their colour estimate, throughout the entire stimulus presentation. This manipulation is crucial for equating sustained attention and working memory demands across the time and colour tasks since (1) time perception necessitates attention being sustained for the entire duration of the stimulus $(\sim 1$ s.), whereas perception of a static colour would occur within the first hundred milliseconds or so, and (2) time perception requires stimulus onset to be held in working memory and then continually updated as time elapses, whereas perception of a static colour would require no such updating of working memory.

\section{Temporal and spatial orienting of attention}

Task The task manipulated participants' expectations of where or when target stimuli would appear within an experimental display. A central cue (inset Fig. 2) predicted either the location (spatial condition) or onset (temporal
Fig. 2 Temporal and spatialorienting task. Participants detected a target appearing at one of two peripheral locations (left/right $)$ after one of two cue-target intervals (short/long) as quickly as possible. In the spatial or temporal condition, the visual cue predicted where or when (respectively) the target was likely to appear, with $80 \%$ validity. In the neutral condition, the visual cue carried neither spatial nor temporal information

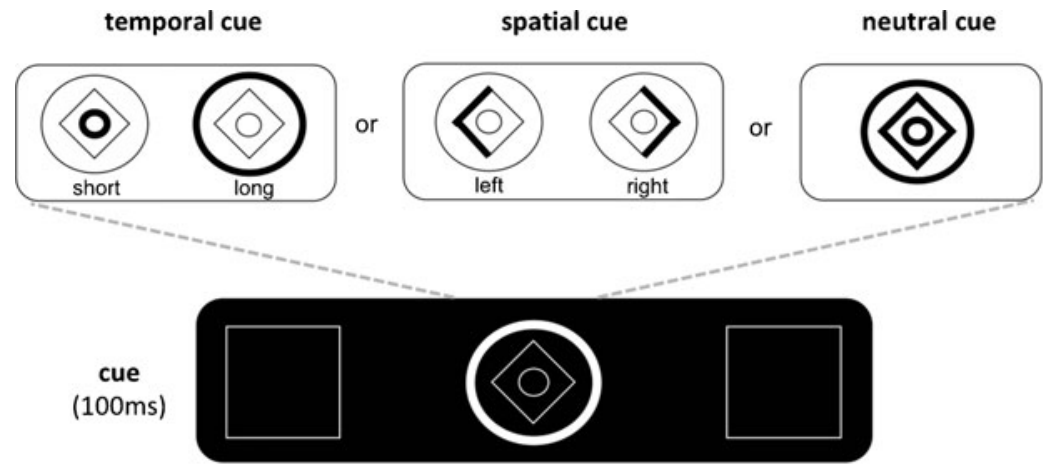

interval (750/1500ms)

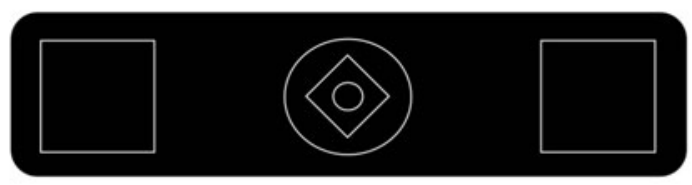

target (100ms)

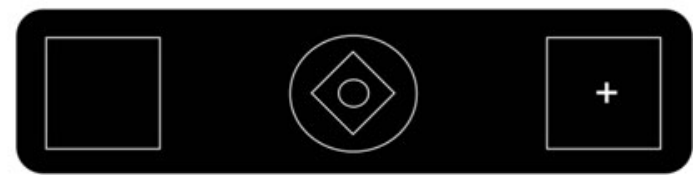


condition) of an imminent target or else it provided neither temporal nor spatial information (neutral condition). In the spatial and temporal conditions, cues could either correctly (valid trials) or incorrectly (invalid trials) predict where or when the target would appear, with an overall ratio of 80:20 valid/invalid trials. In the neutral conditions, the participants could predict neither where nor when the target would appear. The participants' task in all three conditions was simply to detect the target as rapidly as possible, while avoiding mistakes. Participants indicated covert detection of the target by pressing a response button with their right index finger. Inter-trial intervals varied between 600 and $1,000 \mathrm{~ms}$. The computer recorded reaction times to target stimuli. Subjects were thoroughly familiarized with the cues prior to performing 120 experimental trials of each of the three conditions (360 in total). For the temporal and spatial conditions, this corresponded to 96 valid trials and 24 invalid trials.

Stimuli The basic visual display consisted of a central cueing stimulus $\left(1^{\circ}\right.$ eccentricity) and two peripheral boxes ( $7^{\circ}$ eccentricity), inside which the target (' $x$ ' or ' + ') appeared (Fig. 2). The central cue was a compound stimulus consisting of a diamond and two concentric circles. One part of the cue was briefly $(100 \mathrm{~ms})$ highlighted to inform the subject whether to attend to the location or time of onset of the target. During the spatial condition, the left (or right) side of the diamond brightened to inform the subject that the target was likely to appear in the left (or right) peripheral box. During the temporal condition, brightening of the inner or outer circle indicated that the target would appear after a short (750 $\mathrm{ms})$ or long $(1,500 \mathrm{~ms})$ delay, respectively. During the neutral-cue condition, the entire cue brightened, providing no spatial or temporal information. Targets appeared for $100 \mathrm{~ms}$ in one of the two peripheral boxes and after one of two time intervals, according to the nature of the cue: in the spatial condition, targets appeared randomly at the short or long time delay, but their spatial location was determined by the nature of the preceding cue (left/right; valid/invalid); in the temporal condition, targets appeared randomly in the left or right box but their temporal onset was determined by the nature of the preceding cue (short/long; valid/invalid). Spatial locations and temporal onsets were counterbalanced across trials for all three experimental conditions.

\section{Subjective rating scales}

Subjective experience induced by the drug was recorded using a series of clinician-administered questionnaires: the Clinician-Administered Dissociative States Scales
(CADSS, Bremner et al. 1998), Brief Psychiatric Rating Scale, Startup and Startup and a Rating Scale for Psychiatric Symptoms. The time perception subscale of the CADSS (Krystal et al. 1994; 1998) indexed the subjective experience of time more specifically (see Appendix).

\section{Data analysis}

Temporal and colour discrimination

Mean accuracy (percentage of trials correct), omissions (percentage of missed responses) and reaction times (RTs) were analysed in $2 \times 2 \times 2$ repeated measures ANOVAs, with task (time/colour) and treatment (ketamine/placebo) as within-subjects factors, and treatment order (ketamineplacebo/placebo-ketamine) as a between-subjects factor. In addition, proportion of under or overestimates in the time condition were analysed in a $2 \times 3 \times 2 \times 2$ repeated measures ANOVA, with error type (under/overestimate), trial type (probe shorter/equal to/longer than sample) and treatment as within-subjects factors, and treatment order as a between-subjects factor.

Temporal and spatial orienting of attention

Mean RTs for temporal and spatial orienting conditions were analysed in $2 \times 2 \times 2 \times 2$ repeated measures ANOVAs with treatment, cue validity (valid/invalid) and either target onset (short/long) or target location (left/right) (for the temporal and spatial conditions, respectively) as withinsubject factors and treatment order as a between-subject factor. Mean RTs for the neutral condition were analysed in a $2 \times 2 \times 2 \times 2$ repeated measures ANOVA with target onset, target location and treatment as within-subject factors and treatment order as a between-subject factor.

Subjective rating scales

Overall CADSS and CADSS time perception subscale scores were analysed in separate $2 \times 2$ repeated measures ANOVAs with treatment as a within-subject factor and treatment order as a between-subjects factor. Spearman's rank correlations further explored the relationship between these subjective CADSS scores and objective discrimination task performance. Specifically, the association between task performance and (1) global dissociative experience and (2) subjective experience of time was tested by correlating drug-induced change (ketamine-placebo) in mean timing or colour discrimination accuracy with drug-induced change in (1) overall CADSS and (2) CADSS time perception subscale scores, respectively. 


\section{Results}

Temporal and colour discrimination

A significant main effect of task $[F(1,10)=11.80, p<0.01]$ revealed that the colour task was performed less accurately than the timing task. A significant main effect of treatment $[F(1,10)=7.34, p<0.05]$ was qualified by a significant interaction between treatment and task $[F(1,10)=8.73, p<$ 0.05]. Post-hoc analysis with paired $t$ tests indicated that ketamine, as compared to placebo, significantly reduced accuracy in the timing task $(t(11)=3.35, p<0.01)$ but had no effect on performance of the colour task $(t(11)=-0.44$, ns) (Fig. 3a). Ketamine had no significant effect on reaction time or number of omissions. There were no significant main effects of, or interactions with, treatment order.

Given the selective effect of ketamine on timing, we explored these timing data further to examine whether the drug-induced impairments in accuracy were caused by a consistent bias to under or overestimate probe duration. First, each trial was classified according to whether the

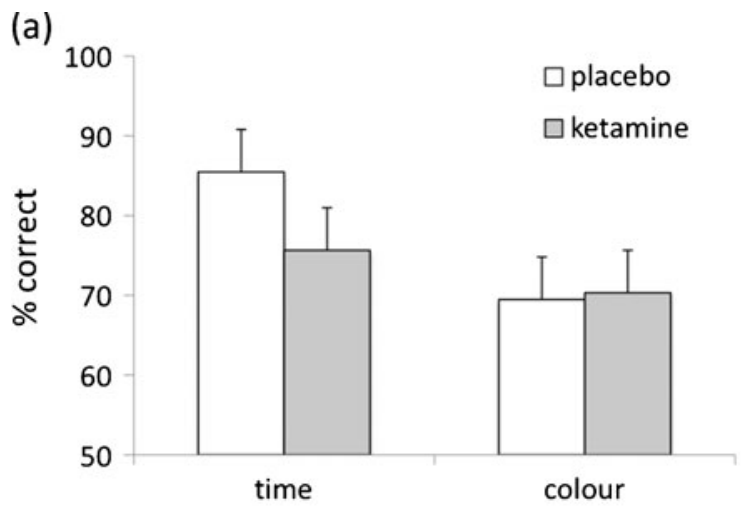

(b)

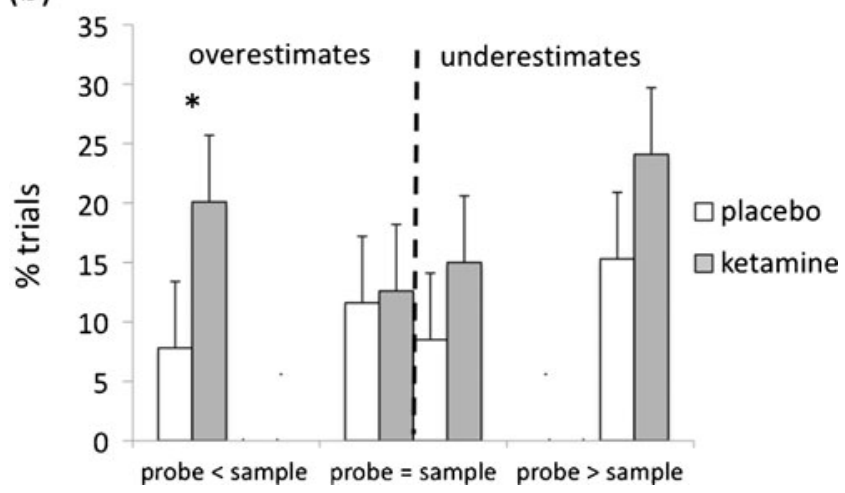

Fig. 3 Ketamine impairs perceptual timing. a Ketamine selectively impaired accuracy (\% correct) of time discrimination, while having no effect on accuracy of colour discrimination. b During the temporal discrimination task, ketamine significantly increased the likelihood (\% trials) that the probe stimulus would be overestimated when the probe was shorter than $(<)$ the sample. Error bars represent the standard error of the mean second stimulus (probe) was longer, shorter or of equal duration to the first stimulus (sample). Then, for each of these three trial types, we calculated the proportion of trials in which a subject under or overestimated the probe compared to the sample. If the probe and sample were of the same duration but the subject judged the probe to be shorter, then this was classed as an underestimate, whereas if the probe was judged to be longer, then this was classed as an overestimate. If the probe was longer than the sample but the participant judged it to be shorter or of equal length, then this was classed as an underestimate. Conversely, if the probe was shorter than the sample but the subject judged it to be longer or of equal length, then this was classed as an overestimate. A main effect of treatment showed that ketamine significantly increased the proportion of both over- and underestimates $[F(1,10)=11.50, p<0.01]$, but did not differentially induce one type of error more than the other (treatment $\times$ error-type interaction $[F(1,10)=0.09$, $n s])$. However, when data were broken down into trial type (probe shorter/equal to/longer than sample), a significant interaction between treatment, error type and trial type $[F(2$, $20)=7.34, p<0.005]$ indicated that ketamine, as compared to placebo, induced an increased incidence of overestimates, particularly when the probe was shorter than the sample $(t(11)=4.14, p<0.005)$ (Fig. 3b). Although there was a corresponding increase in underestimates when the probe was longer than the sample (Fig. 3b), this trend merely approached statistical significance $(t(11)=1.84, p=0.09)$.

Temporal and spatial orienting of attention

Any RTs faster than $100 \mathrm{~ms}$ were considered anticipatory and removed from the analysis. All remaining RTs were log transformed to normalize distributions (resulting RTs nonsignificantly different from a normal distribution as assessed by the Shapiro-Wilks test).

\section{Temporal orienting of attention}

A main effect of cue validity $[F(1,9)=11.54, p<0.01]$ indicated that targets appearing when expected were detected more quickly than targets appearing at an unexpected moment (Table 1). But, as predicted by the results of previous studies using the temporal orienting paradigm (e.g. Coull and Nobre 1998; Coull et al. 2000; Correa et al. 2006), this main effect was qualified by a significant validity $\times$ onset interaction $[F(1,9)=18.24$, $p<0.005]$. Specifically, the benefit of temporally valid cues was significant at the short $(t(10)=-4.71, p=0.001)$ but not long $(\mathrm{t}(10)=-0.48, \mathrm{~ns})$ interval (Table 1$)$ : when a target does not appear after the short interval as expected it must, by process of elimination, appear at the longer one. Such temporal predictability effectively removes the cost of 
targets appearing unexpectedly late. Crucially however, ketamine did not interact with either cue validity $[F(1,9)=$ $0.25, \mathrm{~ns}]$, temporal onset $[F(1,9)=0.06$, ns $]$, nor the interaction between the two $[F(1,9)=0.05$, ns $]$. Nor did ketamine have any overall effect on performance (main effect of drug $[F(1,9)=0.43$, ns $]$ ). Ketamine, therefore, had no effect on the use of temporal cues to predict stimulus onset.

\section{Spatial orienting of attention}

As predicted by prior studies (Posner et al. 1980), a main effect of cue validity $(F(1,9)=29.24, p<0.0001)$ confirmed that targets were detected more quickly when they appeared in expected versus unexpected spatial locations (Table 1). Cue validity did not interact with any other factor, including treatment $[F(1,9)=0.05$, ns $]$. Ketamine, therefore, had no effect on the use of spatial cues to predict stimulus location.

\section{Hazard function}

As predicted by numerous previous studies (see Niemi and Näätänen 1981 for a review), a main effect of onset $[F(1,9)$ $=30.81, p<0.0001]$ confirmed that RTs in the neutral cue condition were significantly faster at long versus short intervals. This speeding effect is due to the increasing conditional probability of target occurrence as a function of the passage of time, i.e. the hazard function, which allows for improved response preparation over time, and thus faster RTs. However, a significant treatment by onset interaction $[F(1,9)=5.83, p<0.05]$ revealed that, as compared to placebo, the relative speeding of RTs at the long interval was attenuated by administration of ketamine (Fig. 4). Therefore, although ketamine did not significantly slow performance in a global manner (main effect of drug $[F(1,9)=1.71$, ns $])$, it selectively compromised the beneficial effects of the hazard function on performance.

Subjective rating scales

Consistent with numerous previous reports (e.g. Krystal et al. 1994, 1998; Fu et al. 2005) a main effect of treatment on the overall CADSS score $[F(1,10)=10.95, p<0.01]$ confirmed that ketamine induced a general dissociative state in healthy volunteers, though this effect tended $[F(1,10)=4.61, p<0.06]$ to be more pronounced in subjects receiving ketamine in the first session and placebo in the second (Table 2). More specifically, ketamine significantly $[F(1,10)=7.22, p<0.05]$ increased scores on the CADSS time perception subscale (see also Krystal et al. 1998), indicating a drug-induced distortion in the subjective experience of time. Again, this effect was greater 


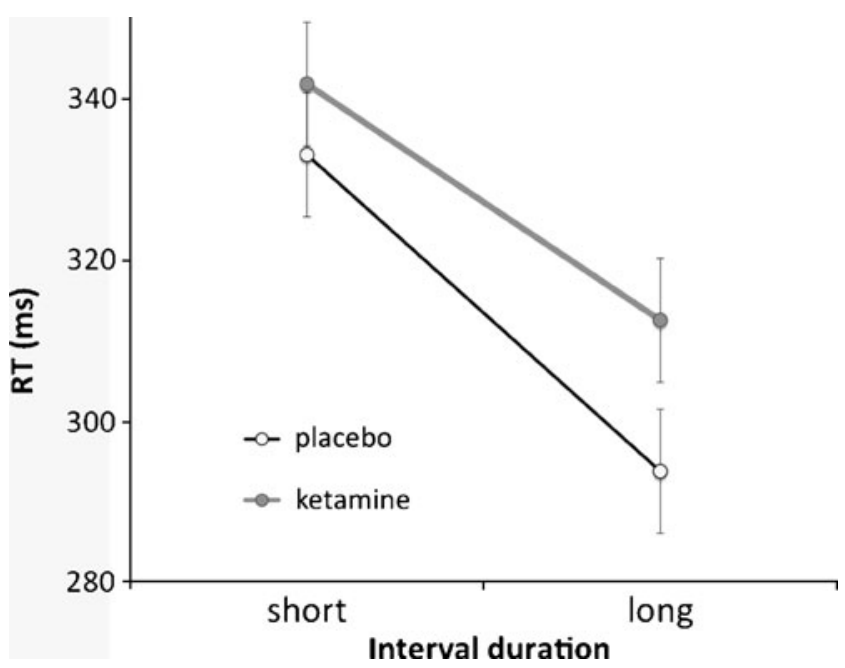

Fig. 4 Ketamine attenuates the beneficial effect of the hazard function. The neutral cue condition of the orienting task produces faster RTs for targets appearing after long rather than short intervals due to the "hazard function". Ketamine slowed RTs selectively at the long, not short, intervals, thus significantly attenuating the benefit that is normally afforded by long delays. Error bars represent the standard error of the mean

$[F(1,10)=5.09, p<0.05]$ in subjects receiving ketamine in the first session and placebo in the second (Table 2).

We used Spearman's rank correlations to search for a link between these subjective measures of perceptual experience and more objective measures of visual perception. Neither the overall CADSS score, nor the time perception subscale, correlated with overall accuracy (\% correct) of the time (Spearman's rho $=-0.02$; Spearman's rho $=0.29$, respectively, ns) or colour (Spearman's rho $=-0.30$; Spearman's rho $=-0.06$ respectively, ns) discrimination task. However, the overall time accuracy score incorporates errors of both over- and underestimates of probe duration. Prior reports of biases to either over(Johnson and Petzel 1971; Tysk 1983) or under- (Rabin 1957; Elvevåg et al. 2003) estimation of duration in patients with schizophrenia prompted us to explore our data further for potential links between the subjective experience of time and the direction of objective temporal error (i.e. proportion of over- or underestimates). We found a significant correlation between scores on the CADSS time perception subscale and the proportion of overestimates (Spearman's rho $=-0.60, p<0.05$ ), though not underestimates (Spearman's rho $=0.25, \mathrm{~ns}$ ) in the time discrimination task.

\section{Discussion}

Administration of an acute dose of ketamine $(100 \mathrm{ng} / \mathrm{ml}$ blood plasma level) selectively impaired perception of temporal, but not colour, attributes of visual stimuli in healthy volunteers. As compared to placebo, ketamine reduced accuracy of perceptual timing while having no effect on accuracy of a colour perception task that was matched to the timing task for accessory cognitive processes, such as visual processing, sustained attention and working memory (WM). This is the first time that selective effects of ketamine on a perceptual timing task have been shown. Despite our relatively small sample size $(n=12)$, the selectivity of the results, demonstrated by a significant task by drug interaction, is striking. Although the colour task was more difficult than the timing task, the lack of drug effect on the colour task is unlikely to merely reflect a floor effect since participants' performance in both sessions ( $70 \%$ accuracy) was well above chance $(33 \%)$, allowing sufficient margin for further deterioration in performance. Selective impairment on performance of the timing task was reflected in participants' subjective assessment of time. Ketamine significantly altered the inner experience of time and drug-induced changes in subjective ratings correlated with objective measures of duration overestimation in the perceptual timing task.

Further evidence of timing disturbances were noted during performance of a cued reaction time (RT) task with variable cue-target intervals. In this task, RTs are generally faster for targets appearing at long, rather than short, intervals due to the increasing conditional probability of target appearance over time (Niemi and Näätänen 1981). However, ketamine slowed RTs selectively at the long interval, thereby attenuating the increase

Table 2 Mean ( \pm standard error) scores on the CADSS index of dissociative experience

\begin{tabular}{lllll}
\hline & \multicolumn{2}{l}{ CADSS overall } & & \multicolumn{2}{l}{ CADSS time perception } \\
\cline { 2 - 3 } & $\mathrm{K} / \mathrm{P}$ & $\mathrm{P} / \mathrm{K}$ & $\mathrm{K} / \mathrm{P}$ & $\mathrm{P} / \mathrm{K}$ \\
\hline Placebo & $0.04( \pm 0.04)$ & $0.10( \pm 0.06)$ & $0.28( \pm 0.18)$ \\
Ketamine & $1.06( \pm 0.39)$ & $0.32( \pm 0.13)$ & $1.28( \pm 0.40)$ & $0.39( \pm 0.18)$
\end{tabular}

Higher scores represent a greater sense of dissociation and disturbed perception. Overall CADSS score represents the average score of all 27 questions, whereas the time perception subscale is calculated as the mean of the three questions relating specifically to the subjective experience of time. Scores are shown as a function of whether the participant received ketamine in the first session and placebo in the second $(\mathrm{K} / \mathrm{P})$ or vice versa $(\mathrm{P} / \mathrm{K})$ 
in speed that is normally seen at long delays. By contrast, ketamine had no significant effect on RT when temporal pre-cues could be used to predict in advance when the target would appear. Similarly, ketamine had no effect on the ability to use spatial cues to predict target location, consistent with previous results in schizophrenic patients (Carter et al. 1992).

Are effects of ketamine on timing due to WM deficits?

Rammsayer (2006) concluded that effects of the NMDA receptor antagonist memantine on timing in the seconds $(\sim 1 \mathrm{~s}$.) but not tens of milliseconds $(\sim 50 \mathrm{~ms})$ range were due to a primary effect on the additional WM processes that are needed to process seconds-range durations. We aimed to disentangle drug effects on timing from effects on WM by examining the effects of ketamine on two tasks that were matched for WM demands, in terms of both manipulation and maintenance in WM. During the 500-1,500 ms stimulus presentation time in both tasks, subjects had to maintain attention and integrate (i.e. manipulate) either duration or colour information in WM: for the timing task, duration estimates had to be updated incrementally throughout stimulus presentation; for the colour task, colour estimates had to be averaged across stimulus presentation. Both tasks also required that a sample stimulus be stored (i. e. maintained) in WM for a delay of $2-4 \mathrm{~s}$ for comparison with a later probe, and for the resulting decision to be maintained in WM until appearance of the response screen. Since mnemonic (and attentional) load were thereby matched across tasks, selective effects of ketamine on timing, but not colour, discrimination, suggests that ketamine-induced impairments do not simply reflect a functional side effect of the drug on the WM processes that are necessary for processing seconds range rather than millisecond timing.

However, one difference between the timing and colour task was the specific way in which information was manipulated in WM. For the timing task, information was incrementally accumulated, whereas for the colour task, it was averaged. Accumulation implies a unidirectionality, a fundamental feature of the flow of time itself ('time's arrow', Eddington 1928). Averaging does not imply this unidirectionality. It is possible therefore, that ketamine influences timing behaviour by selectively impairing the ability to manipulate information in WM in a particular direction or order. A related possibility is that subjects were accumulating not time, but a numerical count of the flickering stimulus. However, this is unlikely given that (1) each flicker was presented for a duration of only $80 \mathrm{~ms}$ necessitating an abnormally fast rate of counting and (2) the vast majority of our trials contained at least one stimulus whose duration was below the critical 1,200 ms value at which counting begins to become a useful strategy (Grondin et al. 1999).

Temporal expectations and the flow of time

As expected, both spatial (Posner et al. 1980) and temporal (Coull and Nobre 1998) cues speeded RTs when targets appeared at expected, rather than unexpected, locations or delays, respectively. These RT effects were not modulated by ketamine, in either the spatial or temporal domain. Although the glutamatergic system has previously been implicated in signaling mismatch between expected and actual outcome (also known as the prediction error) (Corlett et al. 2007a; 2011), we found no evidence in this study for a selective effect of ketamine on RTs to unexpected target locations or onsets. This null effect could represent a lack of effect of ketamine on the processing of unexpected events. Alternatively, it may represent the insensitivity of our behavioural measures: the behavioural consequences of prediction error are rather elusive, and it has previously proven easier to demonstrate the underlying neural responses to prediction error, and their modulation by ketamine, using, for example electrophysiological recordings (e.g. Schultz 1998; Umbricht et al. 2002) or fMRI (e.g. McClure et al. 2003; O'Doherty et al. 2003; Corlett et al. 2006). Future studies examining the effects of ketamine on neural responses to unexpected locations or onsets could help resolve this issue. A final possibility is that these null effects simply reflect a lack of power in the data. However, the predicted behavioural advantages of valid versus invalid cues were clearly present in both the temporal and spatial conditions, itself suggestive of sufficient power. Moreover, although ketamine had no effect on speed of responding in the temporal or spatial conditions, it selectively slowed RTs to long delay targets in the neutral condition. Together, these patterns of data suggest a selectivity of effect rather than insufficient power.

In the orienting task, temporal cues allow subjects to predict event onset, thus generating temporal expectations that help speed performance (Coull and Nobre 1998; Griffin et al. 2001). Yet, even when the onset of the imminent event cannot be predicted by arbitrary, pre-learned cues, the unidirectional flow of time itself (Eddington 1928) provides a degree of predictive power via the hazard function. In our study, the neutral cue condition of the orienting task, in which participants did not know in advance, whether the target would appear at the short or long delay, provides an index of the hazard function. We observed the expected speeding of RTs for targets appearing at long versus short delays following administration of placebo. Ketamine, however, significantly attenuated this increase in response speed (Fig. 4), slowing RTs selectively at the longer delay. Previous studies using a fixed foreperiod paradigm have also 
reported ketamine-induced slowing selectively at long delays (Guillermain et al. 2001; Micallef et al. 2004). This was interpreted as reflecting an effect of ketamine on motor preparation processes due to particularly impaired temporal resolution at long delays. However, this is an unlikely explanation for our own results since ketamine did not slow RTs at long intervals when participants could use arbitrary temporal cues to predict in advance when the target would appear (i.e. the temporal cue condition of the orienting task), suggesting they were perfectly able to estimate and prepare for the long interval as long as it had been cued beforehand. Ketamine did not therefore affect the ability to use prelearned cues to predict in advance when the target would occur but, instead, interfered with the ability to use the very passage of time itself to predict target occurrence when no other sources of temporal information were available. Ketamine effects have previously been interpreted within a Bayesian framework (Corlett et al. 2009; 2011), in which information processing is contingent upon prior expectations. In Bayesian terms, our data show that ketamine does not disrupt the ability to make use of prior expectations to interpret sensory events, but does interfere with the ability to generate expectations online as a function of sensory evidence accumulated over time.

\section{Relevance to schizophrenia}

Temporal predictability in the temporal cue condition of the orienting task is driven by external, visual cues. Conversely, temporal predictability in the neutral cue condition, where visual cues are uninformative, depends upon an internal sense of the passage of time. After administration of ketamine, participants made good use of external visual cues to make temporal predictions, but were less able to use internal estimates of time to make predictions. This psychopharmacological dissociation is reminiscent of findings in patients with schizophrenia who rely more upon external sensory stimuli to interpret the consequences of their actions, rather than internal sensorimotor processes (Synofzik et al. 2010; Voss et al. 2010). Moreover, while the temporal cue condition requires straightforward maintenance of a fixed a priori temporal prediction in WM, the neutral cue condition requires updating (i.e. manipulation) of the prediction as a function of elapsing time. Notably, both ketamine and schizophrenia have previously been associated with deficits in manipulation, rather than maintenance in WM (Fletcher and Honey 2006). Our orienting data, taken together with the results on the temporal discrimination task discussed above, support the proposal that ketamine interferes with the way in which the unidirectional flow of time is integrated within WM. Intriguingly, disturbances in the temporal flow (pastpresent-future) of causal experience have previously been suggested to underpin certain symptoms of schizophrenia (Fuchs 2007). The timing deficits observed in schizophrenia may therefore be more accurately characterised as impairments in temporal flow, such that the beginning, middle and end of the event to be timed are not necessarily processed (or retained in $\mathrm{WM}$ ) in the correct order, thus compromising duration estimates. Further experiments are clearly needed to test this hypothesis more directly.

Patients with schizophrenia have previously been reported to both over- (Johnson and Petzel 1971; Tysk 1983) and underestimate (Rabin 1957; Elvevåg et al. 2003) stimulus duration. We therefore examined whether ketamine produced a consistent temporal error in one direction or the other by measuring the incidence of over- or underestimation of probe duration. Overall, ketamine induced both types of error, with no significant bias in one direction or the other (see also Cheng et al. 2006). However, a particularly high incidence of drug-induced overestimation was evident whenever the probe stimulus was shorter than the sample. This result is unlikely to reflect a ketamineinduced increase in speed of an internal clock (Gibbon et al. 1984), because any change in clock speed would apply equally to both sample and probe stimuli. Instead, ketamine appears to have either selectively elongated perception of the current (probe) event, or contracted the memorized representation of the sample. This issue could be addressed by future experiments in which the sample duration is encoded either before or after administration of ketamine (see Malapani et al. (2002) for an example of this approach in patients with Parkinson's disease).

Finally, patients with schizophrenia (Carroll et al. 2008) or individuals at high risk for schizophrenia (Penney et al. 2005) have previously been reported to show larger than normal differences in the relative accuracy of auditory versus visual timing, potentially due to a preferential impairment of timing in the auditory modality (Carroll et al. 2008). If ketamine can be considered a useful model of schizophrenia, then we may expect to replicate this 'modality effect' with ketamine and, furthermore, to predict that its effects on timing would be even more evident when tested in the auditory domain.

\section{Possible neural substrates}

We have shown that ketamine impairs accuracy on a temporal discrimination task that has previously been shown to engage Supplementary Motor Area, right prefrontal cortex and basal ganglia (Coull et al. 2004; 2008). Although ketamine had no effect on the ability to use visual cues to predict stimulus onset in the temporal orienting task, a process previously shown to engage left inferior parietal cortex and intraparietal sulcus (Coull and Nobre 1998; Coull et al. 2011), it did impair the ability to use the 
flow of time to make temporal predictions (the hazard function), a process that has consistently been shown to depend upon right prefrontal cortex (Vallesi et al. 2007a, 2007b, 2009; Bueti et al. 2010). Taken together with data from the temporal discrimination task, we suggest that ketamine may impair timing by modulating right prefrontal cortex function. This hypothesis is supported by functional imaging data showing that ketamine modulates activity in prefrontal cortex, among other areas, during tasks of WM and executive function (Fu et al. 2005; Honey et al. 2008) or prediction error (Corlett et al. 2006).

These data might indicate therefore that timing depends upon glutamatergic function in prefrontal cortex. Alternatively, ketamine is known to increase prefrontal dopamine in rats (Bowers and Morton 1994; Verma and Moghaddam 1996), suggesting that its effect on timing may instead be a result of drug-induced modulation of prefrontal dopamine. Therefore, just as dysregulation of dopaminergic innervation of prefrontal cortex has been suggested as the common underlying mechanism for both schizophrenia and ketamine-induced impairments in WM (Fletcher and Honey 2006), it may also explain the timing deficits associated with these two states. Notably, the dopaminergic system has repeatedly been implicated in timing (Meck 1996), with converging evidence from the animal and clinical literatures (Coull et al. 2011). Yet dopaminergic manipulations provoke consistent changes in clock speed, with enhanced dopaminergic activity leading to overestimates of stimulus duration (Maricq and Church 1983; Meck 1983; Matell et al. 2004, 2006) and reduced activity inducing underestimates (Maricq and Church 1983; Meck 1983, 1986; MacDonald and Meck 2005) and results of the present study, as well as prior work in rats (Cheng et al. 2006, 2007a), suggest ketamine has no effect on clock speed. Instead, Cheng et al. (2007a, 2007b) suggest ketamine 'unlocks' the clock-speeding effects of dopaminergic agonists that are otherwise attenuated by extensive training. Specifically, although administration of dopaminergic agonists is known to speed the internal clock, extensive training on the task abolishes this effect (Cheng et al. 2007a, 2007b). The hypothesis is that as performance on the timing task becomes more and more automated through training, the dopaminergic system becomes less critical for timing, and instead, the glutamatergic system takes over to mediate a more habitual form of timing behaviour. If a glutamatergic antagonist, such as ketamine, is administered, then control reverts to the dopaminergic system, meaning dopaminergic agents will once more produce their clockspeeding effects. So why, in contrast to Cheng et al. (2006, 2007a), does ketamine modulate timing in our relatively naive participants? The answer may lie, once more, with the hazard function. Keeping track of the unidirectional flow of time in WM in order to time current events, as well as to predict the onset of future events, is an overlearned, automated process that, as suggested above, appears to be subject to disruption by ketamine. Therefore, the results of this study appear not to reflect a dopamine-dependent clock-speed effect, but may instead indicate a glutamate-dependent modulation of the unidirectional nature of 'time's arrow'. Alternatively, in line with ketamine's effects on the manipulation of information in WM (Honey et al. 2003, 2004), our results may reflect dopamine-dependent modulation of the way in which temporal information is integrated within WM. Future studies combining functional neuroimaging and psychopharmacological methodologies could ascertain more directly the role of prefrontal dopamine or glutamate in the effects of ketamine on timing or, more specifically, on the ability to integrate the unidirectional flow of time in WM.

Acknowledgements PCF is funded by the Wellcome Trust and the Bernard Wolfe Health Neuroscience Fund. This work was completed within the Wellcome Trust Clinical Research Facility, Cambridge and was supported by the joint Wellcome- and MRC-funded Behavioural and Clinical Neuroscience Institute. The experiment complies with current UK laws.

Disclosure PCF has received funds from GSK for consultation services and from Astra Zeneca for a lecture.

Open Access This article is distributed under the terms of the Creative Commons Attribution Noncommercial License which permits any noncommercial use, distribution, and reproduction in any medium, provided the original author(s) and source are credited.

\section{Appendix}

The Clinician Administered Dissociative States Scales (CADSS, Bremner et al. 1998) comprises 19 self-report questions and 8 observer ratings, rated from 0 (not at all) to 4 (extremely), and incorporates five subscales (Krystal et al. 1994): body perception, environmental perception, feelings of unreality, memory impairment and time perception. The overall CADSS score (mean response to all questions) provides a global measure of dissociative experiences, while the time perception subscale (Krystal et al. 1994; 1998) indexes the subjective experience of time more specifically by averaging the three CADSS questions that relate directly to the perception of time, namely:

Q1: Do things seem to be moving in slow motion?

Q12: Does this experience seem to take much longer than you would have expected?

Q13: Do things seem to be happening very quickly, as if there is a lifetime in a moment? 


\section{References}

Absalom AR, Lee M, Menon DK, Sharar SR, De Smet T, Halliday J et al (2007) Predictive performance of the Domino, Hijazi, and Clements models during low-dose target-controlled ketamine infusions in healthy volunteers. Br J Anaesth 98:615-623

Bowers MB Jr, Morton JB (1994) Regional brain catecholamines and metabolites following THC, PCP and MK-801. Prog Neuropsychopharmacol Biol Psychiatry 18:961-964

Bremner JD, Krystal JH, Putnam FW, Southwick SM, Marmar C, Charney DS, Mazure CM (1998) Measurement of dissociative states with the Clinician-Administered Dissociative States Scale (CADSS). J Trauma Stress 11:125-136

Bueti D, Bahrami B, Walsh V, Rees G (2010) Encoding of temporal probabilities in the human brain. J Neurosci 30:4343-4352

Buonomano DV, Bramen J, Khodadadifar M (2009) Influence of the interstimulus interval on temporal processing and learning: testing the state-dependent network model. Philos Trans R Soc Lond B Biol Sci 364:1865-1873

Carroll CA, Boggs J, O'Donnell BF, Shekhar A, Hetrick WP (2008) Temporal processing dysfunction in schizophrenia. Brain Cogn 67:150-161

Carroll CA, O'Donnell BF, Shekhar A, Hetrick WP (2009) Timing dysfunctions in schizophrenia as measured by a repetitive finger tapping task. Brain Cogn 71:345-353

Carter CS, Robertson LC, Chaderjian MR, Celaya LJ, Nordahl TE (1992) Attentional asymmetry in schizophrenia: controlled and automatic processes. Biol Psychiatry 31:909-918

Cheng RK, MacDonald CJ, Meck WH (2006) Differential effects of cocaine and ketamine on time estimation: implications for neurobiological models of interval timing. Pharmacol Biochem Behav 85:114-122

Cheng RK, Ali YM, Meck WH (2007a) Ketamine 'unlocks' the reduced clock-speed effect of cocaine following extended training: evidence for dopamine-glutamate interactions in timing and time perception. Neurobiol Learn Mem 88:149-159

Cheng RK, Hakak OL, Meck WH (2007b) Habit formation and the loss of control of an internal clock: inverse relationship between the level of baseline training and the clock-speed enhancing effects of methamphetamine. Psychopharmacology 193:351-362

Corlett PR, Honey GD, Aitken MR, Dickinson A, Shanks DR et al (2006) Frontal responses during learning predict vulnerability to the psychotogenic effects of ketamine: linking cognition, brain activity, and psychosis. Arch Gen Psychiatry 63:611-621

Corlett PR, Honey GD, Fletcher PC (2007a) From prediction error to psychosis: ketamine as a pharmacological model of delusions. J Psychopharmacol 21:238-252

Corlett PR, Murray GK, Honey GD, Aitken MR, Shanks DR, Robbins TW, Bullmore ET, Dickinson A, Fletcher PC (2007b) Disrupted prediction-error signal in psychosis: evidence for an associative account of delusions. Brain 130:2387-2400

Corlett PR, Frith CD, Fletcher PC (2009) From drugs to deprivation: a Bayesian framework for understanding models of psychosis. Psychopharmacology 206:515-530

Corlett PR, Honey GD, Krystal JH, Fletcher PC (2011) Glutamatergic model psychoses: prediction error, learning, and inference. Neuropsychopharmacology 36:294-315

Correa A, Lupiáñez J, Tudela P (2006) The attentional mechanism of temporal orienting: determinants and attributes. Exp Brain Res 169:58-68

Coull JT, Nobre AC (1998) Where and when to pay attention: the neural systems for directing attention to spatial locations and to time intervals as revealed by both PET and fMRI. J Neurosci 18:7426-7435
Coull J, Nobre A (2008) Dissociating explicit timing from temporal expectation with fMRI. Curr Opin Neurobiol 18:137-144

Coull JT, Frith CD, Buchel C, Nobre AC (2000) Orienting attention in time: behavioural and neuroanatomical distinction between exogenous and endogenous shifts. Neuropsychologia 38:808-819

Coull JT, Vidal F, Nazarian B, Macar F (2004) Functional anatomy of the attentional modulation of time estimation. Science 303:15061508

Coull JT, Nazarian B, Vidal F (2008) Timing, storage, and comparison of stimulus duration engage discrete anatomical components of a perceptual timing network. J Cogn Neurosci 20:2185-2197

Coull JT, Cheng R-K, Meck WH (2011) Neuroanatomical and neurochemical substrates of timing. Neuropsychopharmacology 36:3-25

Duncan EJ, Madonick SH, Parwani A, Angrist B, Rajan R, Chakravorty S, Efferen TR, Szilagyi S, Stephanides M, Chappell PB, Gonzenbach S, Ko GN, Rotrosen JP (2001) Clinical and sensorimotor gating effects of ketamine in normals. Neuropsychopharmacology 25:72-83

Davalos DB, Kisley MA, Ross RG (2003) Effects of interval duration on temporal processing in schizophrenia. Brain Cogn 52:295301

Eddington AS (1928) The nature of the physical world. Cambridge University Press, Cambridge

Elvevåg B, McCormack T, Gilbert A, Brown GD, Weinberger DR, Goldberg TE (2003) Duration judgements in patients with schizophrenia. Psychol Med 33:1249-1261

Elithorn A, Lawrence C (1955) Central inhibition: some refractory observations. Quart J Exp Psychol 11:211-220

Fletcher PC, Honey GD (2006) Schizophrenia, ketamine and cannabis: evidence of overlapping memory deficits. Trends Cogn Sci 10:167-174

Freedman BJ (1974) The subjective experience of perceptual and cognitive disturbances in schizophrenia. A review of autobiographical accounts. Arch Gen Psychiatry 30:333-340

Fu CH, Abel KM, Allin MP, Gasston D, Costafreda SG, Suckling J, Williams SC, McGuire PK (2005) Effects of ketamine on prefrontal and striatal regions in an overt verbal fluency task: a functional magnetic resonance imaging study. Psychopharmacology 183:92-102

Fuchs T (2007) The temporal structure of intentionality and its disturbance in schizophrenia. Psychopathology 40:229-235

Gibbon J, Church RM, Meck WH (1984) Scalar timing in memory. Ann NY Acad Sci 423:52-77

Gibbon J, Malapani C, Dale CL, Gallistel C (1997) Toward a neurobiology of temporal cognition: advances and challenges. Curr Opin Neurobiol 7:170-184

Griffin IC, Miniussi C, Nobre AC (2001) Orienting attention in time. Front Biosci 6:d660-d671

Grondin S, Meilleur-Wells G, Lachance R (1999) When to start explicit counting in a time-intervals discrimination task: a critical point in the timing process by humans. J Exp Psychol Hum Percept Perform 25:993-1004

Guillermain Y, Micallef J, Possamaï C, Blin O, Hasbroucq T (2001) $\mathrm{N}$-methyl-D-aspartate receptors and information processing: human choice reaction time under a subanaesthetic dose of ketamine. Neurosci Lett 303:29-32

Honey RA, Turner DC, Honey GD, Sharar SR, Kumaran D, PomarolClotet E, McKenna P, Sahakian BJ, Robbins TW, Fletcher PC (2003) Subdissociative dose ketamine produces a deficit in manipulation but not maintenance of the contents of working memory. Neuropsychopharmacology 28:2037-2044

Honey RA, Honey GD, O'Loughlin C, Sharar SR, Kumaran D, Bullmore ET et al (2004) Acute ketamine administration alters 
the brain responses to executive demands in a verbal working memory task: an FMRI study. Neuropsychopharmacology 29:1203-1214

Honey GD, Corlett PR, Absalom AR, Lee M, Pomarol-Clotet E et al (2008) Individual differences in psychotic effects of ketamine are predicted by brain function measured under placebo. J Neurosci 28:6295-6303

Johnson JE, Petzel TP (1971) Temporal orientation and time estimation in chronic schizophrenics. J Clin Psychol 27:194-196

Kim J, Glahn DC, Nuechterlein KH, Cannon TD (2004) Maintenance and manipulation of information in schizophrenia: further evidence for impairment in the central executive component of working memory. Schizophr Res 68:173-187

Krystal JH, Karper LP, Seibyl JP, Freeman GK, Delaney R et al (1994) Subanesthetic effects of the noncompetitive NMDA antagonist, ketamine, in humans psychotomimetic, perceptual, cognitive, and neuroendocrine responses. Arch Gen Psychiatry 51:199-214

Krystal JH, Karper LP, Bennett A, D'Souza DC, Abi-Dargham A et al (1998) Interactive effects of subanesthetic ketamine and subhypnotic lorazepam in humans. Psychopharmacology 135:213229

Krystal JH, Abi-Saab W, Perry E, D'Souza DC, Liu N et al (2005) Preliminary evidence of attenuation of the disruptive effects of the NMDA glutamate receptor antagonist, ketamine, on working memory by pretreatment with the group II metabotropic glutamate receptor agonist, LY354740, in healthy human subjects. Psychopharmacology 179:303-309

Lhamon WT, Goldstone S (1956) The time sense: estimation of one second durations by schizophrenic patients. AMA Arch Neurol Psychiatry 76:625-629

Luce RD (1986) Response times: their role in inferring elementary mental organization. Oxford University Press, New York

MacDonald CJ, Meck WH (2005) Differential effects of clozapine and haloperidol on interval timing in the supraseconds range. Psychopharmacology 182:232-244

McClure SM, Berns GS, Montague PR (2003) Temporal prediction errors in a passive learning task activate human striatum. Neuron 38:339-346

Malapani C, Deweer B, Gibbon J (2002) Separating storage from retrieval dysfunction of temporal memory in Parkinson's disease. J Cogn Neurosci 14:311-322

Malhotra AK, Pinals DA, Weingartner H, Sirocco K, Missar CD et al (1996) NMDA receptor function and human cognition: the effects of ketamine in healthy volunteers. Neuropsychopharmacology 14:301-307

Maricq AV, Church RM (1983) The differential effects of haloperidol and methamphetamine on time estimation in the rat. Psychopharmacology 79:10-15

Matell MS, King GR, Meck WH (2004) Differential modulation of clock speed by the administration of intermittent versus continuous cocaine. Behav Neurosci 118:150-156

Matell MS, Bateson M, Meck WH (2006) Single-trial analyses demonstrate that increases in clock speed contribute to the methamphetamine-induced horizontal shifts in peak-interval timing functions. Psychopharmacology 188:201-212

Meck WH (1983) Selective adjustment of the speed of internal clock and memory processes. J Exp Psychol Anim Behav Process 9:171-201

Meck WH (1986) Affinity for the dopamine D2 receptor predicts neuroleptic potency in decreasing the speed of an internal clock. Pharmacol Biochem Behav 25:1185-1189

Meck WH (1996) Neuropharmacology of timing and time perception. Cognit Brain Res 3:227-242

Micallef J, Gavaudan G, Burle B, Blin O, Hasbroucq T (2004) A study of a topiramate pre-treatment on the effects induced by a subanaesthetic dose of ketamine on human reaction time. Neurosci Lett 369:99-103

Michon JA (1985) The complete time experience. In: Michon JA, Jackson JL (eds) Time, mind, and behavior. Springer, Berlin, pp $21-52$

Morgan CJ, Curran HV (2006) Acute and chronic effects of ketamine upon human memory: a review. Psychopharmacology 188:408 424

Niemi P, Näätänen R (1981) Foreperiod and simple reaction time. Psychol Bull 89:133-162

Nobre AC (2001) Orienting attention to instants in time. Neuropsychologia 39:1317-1328

O'Doherty JP, Dayan P, Friston K, Critchley H, Dolan RJ (2003) Temporal difference models and reward-related learning in the human brain. Neuron 38:329-337

Penney TB, Meck WH, Roberts SA, Gibbon J, Erlenmeyer-Kimling L (2005) Interval-timing deficits in individuals at high risk for schizophrenia. Brain Cognit 58:109-118

Perry W, Heaton RK, Potterat E, Roebuck T, Minassian A, Braff DL (2001) Working memory in schizophrenia: transient "online" storage versus executive functioning. Schizophr Bull 27:157176

Pomarol-Clotet E, Honey GD, Murray GK, Corlett PR, Absalom AR et al (2006) Psychological effects of ketamine in healthy volunteers. Phenomenological study. Br J Psychiatry 189:173-179

Pöppel E (1997) A hierarchical model of temporal perception. Trends Cognit Sci 1:56-61

Posner MI, Snyder C, Davidson BJ (1980) Attention and the detection of signals. J Exp Psychol 109:160-174

Rabin AI (1957) Time estimation of schizophrenics and nonpsychotics. J Clin Psychol 13:88-90

Rammsayer TH (2006) Effects of pharmacologically induced changes in NMDA receptor activity on human timing and sensorimotor performance. Brain Res 1073-1074:407-416

Reutimann J, Yakovlev V, Fusi S, Senn W (2004) Climbing neuronal activity as an event-based cortical representation of time. J Neurosci 24:3295-3303

Schultz W (1998) Predictive reward signal of dopamine neurons. J Neurophysiol 80:1-27

Seeman MV (1976) Time and schizophrenia. Psychiatry 39:189-195

Stone JM, Pilowsky LS (2006) Psychopathological consequences of ketamine. Br J Psychiatry 189:565-566

Synofzik M, Thier P, Leube DT, Schlotterbeck P, Lindner A (2010) Misattributions of agency in schizophrenia are based on imprecise predictions about the sensory consequences of one's actions. Brain 133:262-271

Treisman M (1963) Temporal discrimination and the indifference interval: implications for a model of the 'internal clock'. Psychol Monogr 77:1-31

Tysk L (1983) Time estimation by healthy subjects and schizophrenic patients: a methodological study. Percept Mot Skills 56:983988

Umbricht D, Koller R, Vollenweider FX, Schmid L (2002) Mismatch negativity predicts psychotic experiences induced by NMDA receptor antagonist in healthy volunteers. Biol Psychiatry $51: 400-406$

Vallesi A, Mussoni A, Mondani M, Budai R, Skrap M, Shallice T (2007a) The neural basis of temporal preparation: insights from brain tumor patients. Neuropsychologia 45:2755-2763

Vallesi A, Shallice T, Walsh V (2007b) Role of the prefrontal cortex in the foreperiod effect: TMS evidence for dual mechanisms in temporal preparation. Cereb Cortex 17:466-474

Vallesi A, McIntosh AR, Shallice T, Stuss DT (2009) When time shapes behavior: fMRI evidence of brain correlates of temporal monitoring. J Cognit Neurosci 21:1116-1126 
Verma A, Moghaddam B (1996) NMDA receptor antagonists impair prefrontal cortex function as assessed via spatial delayed alternation performance in rats: modulation by dopamine. J Neurosci 16:373-379

Vogeley K, Kupke C (2007) Disturbances of time consciousness from a phenomenological and a neuroscientific perspective. Schizophr Bull 33:157-165
Voss M, Moore J, Hauser M, Gallinat J, Heinz A, Haggard P (2010) Altered awareness of action in schizophrenia: a specific deficit in predicting action consequences. Brain 133:31043112

Zakay D, Block RA (1996) The role of attention in time estimation processes. In: Pastor MA, Artieda J (eds) Time, internal clocks and movement. Elsevier Sciences, New York, pp 143-164 